\title{
CARACTERÍSTICAS DE CRIANÇAS ONCOLÓGICAS COM FLEBITE EM DISPOSITIVOS INTRAVASCULARES PERIFÉRICOS
}

\author{
Fernanda da Silva Passos ${ }^{1}$; Luciano Marques dos Santos ${ }^{2}$; Paula Horrana Carvalho \\ Silvar $^{3}$ e Larissa Freitas Cerqueira ${ }^{4}$ \\ 1. Voluntária, Graduanda em Enfermagem, Universidade Estadual de Feira de Santana, e-mail: psilvananda@gmail.com \\ 2. Orientador, Departamento de Saúde, Universidade Estadual de Feira de Santana, e-mail: lucmarxenfo@ yahoo.com.br \\ cerqueirafreitaslarissa@gmail.com \\ 3. Participante do Nudes, Departamento de Saúde, Universidade Estadual de Feira de Santana, e-mail: \\ paulahorrana.uefs@yahoo.com.br \\ 4. Participante do Nudes, Departamento de Saúde, Universidade Estadual de Feira de Santana, e-mail: \\ cerqueirafreitaslarissa@gmail.com
}

PALAVRAS-CHAVE: Enfermagem neonatal; terapia infusional; egurança do paciente.

\section{INTRODUÇÃO}

O câncer infanto juvenil é considerado uma doença rara, onde o tratamento da criança e adolescente pode ser realizado em ambientes ambulatoriais, a depender de seu diagnóstico e condição clínica. Independentemente do local, oxistem três formas principais de tratamento para o câncer: quimioterapia, radioterapia e cirurgia, dentre estas modalidades terapêuticas destaca-se a quimioterapia.

No tratamento quimioterápico, a via intravenosa é a mais utilizada, pois a mesma permite a administração da maioria dos agentes quimioterápicos em bolus, por gotejamento e por infusão contínua, através de acesso venoso periférico ou central (Maia et al., 2010). Porém o uso constante desta via pode acarretar danos à rede venosa, a exemplo da flebite, que é definidas como uma inflamação na veia.

Sendo assim, esse estudo possui como objetivo analisar a associação entre características clínicas e sociodemográficas da criança e adolescente, da CIP atual e TIV utilizada com a ocorrência de flebites em crianças e adolescentes hospitalizadas na unidade de oncologia do Hospital Estadual da Criança em Feira de Santana, Bahia.

\section{METODOLOGIA (ou equivalente)}

Trata-se de um estudo transversal em que foi estudada uma amostra por conveniência de 311 punções, das quais 14 apresentaram flebite na unidade de clínica oncológica de um hospital pediátrico de Feira de Santana, Bahia no período de abril de 2015 a dezembro de 2016. A população do estudo foi composta por todas as crianças e adolescentes hospitalizados no referido hospital no período da coleta.

Os dados foram tabulados no SPSS, versão 22.0, e as variáveis descritas em frequências absolutas e relativas. As odds ratio bruta e ajustada foram calculadas através da regressão logística, com intervalo de confiança de 95\%. Empregou-se o Qui-quadrado de Pearson e teste exato de Fisher com nível de significância de 5\% (p<0,05). Este estudo respeitou a Resolução 466/2012 do Conselho Nacional de Saúde.

\section{RESULTADOS E DISCUSSÃO}

A ocorrência de flebite nas CIP realizadas nas crianças atingiu uma taxa de $4,5 \%$, demonstrando uma prevalência aceitável desta complicação na unidade em estudo, já que, de acordo com a INS, a frequência deste evento deve ser de $5 \%$ ou menos em qualquer população (INS - BRASIL, 2013; INS, 2016).

Segundo o presente estudo, a chance de ocorrer flebite entre as crianças que ficaram internadas por um período maior ou igual a 8 dias é quatro vezes a chance daquelas que ficaram internados por até 7 dias $(\mathrm{p}=0,022)$, como também, a chance de flebite entre as 
crianças que tiveram TIV prolongada, ou seja, superior a 7 dias, é onze vezes a chance das que não tiveram TIV prolongada $(\mathrm{p}=0,000)$ (Tabela 1$)$.

Segundo Abdul-Hak e Barros (2014) estudo na população adulta, há uma associação estatisticamente significativa dos casos de flebite com o tempo de internação maior do que 18 dias $(\mathrm{p}=0,002)$. Contudo, o tempo de permanência esta relacionado ao risco de infecção da corrente sanguínea. Quanto maior o tempo de utilização da TIV, maior a possibilidade de desenvolvimento de complicações.

O presente estudo mostrou que a chance de flebite entre as crianças/adolescentes que utilizaram a terapia medicamentosa por quatro ou mais dias é quatro vezes a chances das que utilizaram por um período menor $(\mathrm{p}=0,019)$ (Tabela 1). Entretanto, em estudo com crianças foi observado que após o terceiro dia de TIV a uma redução na ocorrência de flebite, priorizando, portanto a permanência do CIP na criança até o termino TIV, a menos que ocorra alguma complicação relacionada à terapia intravenosa (Bagnasco et al., 2011; Jacinto et al., 2014).

Assim, este resultado pode estar associado ao fato de muitas crianças e adolescente apresentarem más condições da veia, associada às condições clínica, como a própria doença de base, todavia, crianças/adolescentes que utilizam uma TIV prolongada podem ter alterações em seus vasos devido ao uso constante dos mesmo.

O presente estudo também apontou que a chance de flebite entre as crianças que utilizaram o cateter intravenoso periférico por um período maior ou igual a 72 horas é cinco vezes a chance daquelas que utilizaram o cateter por um período menor que 72 horas $(\mathrm{p}=0,012)$ (Tabela 1). Em controvérsia com nosso achado, em pesquisa afirmou-se que não há nenhuma evidência conclusiva de benefícios na mudança de cateteres a cada 72 a 96 horas (Webster et al., 2010).

Observou-se também, que a chance de flebite entre as crianças/adolescentes que foram puncionadas com cateter de calibre até 22 gauge, é quatro vezes a chance daquelas que foram puncionadas com calibre de 24 gauge $(\mathrm{p}=0,025)$ (Tabela 1$)$. Todavia, em estudos realizados com crianças afirmou-se que cateteres de menor calibre (24 gauge) estão associados com um aumento significativo do risco de flebite (Jacinto et al., 2014; Tripathi, Kaushik, Singh, 2008).

Deste modo, este resultado pode estar associado ao fato do uso inadequado do calibre do cateter em relação a características da veia da criança/adolescente, sendo assim, cateteres de maior calibre podem produzir uma maior taxa de flebites deve ao fato de exercerem um maior atrito contra a parede do vaso cateterizado, levando a uma flebite mecânica. 
Tabela 1. Associações entre as variáveis sociodemograficas, relacionadas à CIP atual, condições da rede venosa e TIV atual utilizada e a ocorrência de flebite em uma unidade pediátrica no interior da Bahia, Feira de Santana, no período de 2015 a 2016.

\begin{tabular}{|c|c|c|c|c|c|c|c|c|}
\hline \multirow[b]{2}{*}{ Variáveis } & \multicolumn{8}{|c|}{ Flebite } \\
\hline & $\begin{array}{c}\text { Sim } \\
(n=14) \\
N(\%)\end{array}$ & $\begin{array}{c}\text { Não } \\
(\mathbf{n}=297) \\
\mathbf{N}(\%)\end{array}$ & ORb & IC & P-valor & ORa & IC & P-valor \\
\hline $\begin{array}{l}\text { Tempo de } \\
\text { hospitalizaçã } \\
\text { o (dias) }\end{array}$ & & & & & & & & \\
\hline $\begin{array}{l}\text { Maior ou igual } \\
\text { a } 8 \text { dias }\end{array}$ & $5(33,3)$ & $10(66,7)$ & 5,85 & $\begin{array}{l}1,678- \\
20411\end{array}$ & $0,006 *$ & 4,94 & $\begin{array}{l}1,266- \\
19,309\end{array}$ & $\mathbf{0 , 0 2 2} *$ \\
\hline Até 7 dias & $9(3,0)$ & $\begin{array}{l}287 \\
(97,0)\end{array}$ & & & & & & \\
\hline $\begin{array}{l}\text { TIV } \\
\text { prolongada }\end{array}$ & & & & & $0,000 *$ & & & \\
\hline Sim & $5(27,8)$ & $13(72,2)$ & 12,1 & 3,560- & & 11,83 & 3,111- & $0,000 *$ \\
\hline Não & $9(3,1)$ & $\begin{array}{l}284 \\
(96,9)\end{array}$ & & 41,375 & & & 45,020 & \\
\hline 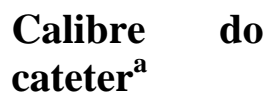 & & & & & & & & \\
\hline $\begin{array}{l}22 \mathrm{G} \\
24 \mathrm{G}\end{array}$ & $\begin{array}{l}11(8,3) \\
3(1,7)\end{array}$ & $\begin{array}{l}122 \\
(91,7) \\
171 \\
(98,3)\end{array}$ & 5,10 & $\begin{array}{l}1,396- \\
18,70\end{array}$ & $0,014 * *$ & 4,99 & $\begin{array}{l}1,225- \\
20,348\end{array}$ & $0,025 * *$ \\
\hline $\begin{array}{ll}\text { Tempo } & \text { de } \\
\text { utilização } & \text { do } \\
\text { CIP } & \end{array}$ & & & & & & & & \\
\hline $\begin{array}{l}\text { Maior ou igual } \\
\text { a } 72\end{array}$ & $4(17,4)$ & $19(82,6)$ & 5,85 & $\begin{array}{l}1,678- \\
20,411\end{array}$ & $0,006 * *$ & 5,50 & $\begin{array}{l}1,456- \\
20,834\end{array}$ & $0,012 * *$ \\
\hline Menor que 72 & $10(3,5)$ & $\begin{array}{l}278 \\
(96,5)\end{array}$ & & & & & & \\
\hline $\begin{array}{l}\text { Tempo de } \\
\text { utilização da } \\
\text { terapia } \\
\text { medicamento } \\
\text { sa (dias) }\end{array}$ & & & & & & & & \\
\hline $\begin{array}{l}\text { Quatro ou } \\
\text { mais }\end{array}$ & $4(15,4)$ & $22(84,6)$ & 5,00 & $\begin{array}{l}1,450- \\
17,247\end{array}$ & $0,011 * *$ & 4,63 & $\begin{array}{l}1,285- \\
16,754\end{array}$ & $0,019 * *$ \\
\hline Até 3 dias & $10(3,5)$ & $\begin{array}{l}275 \\
(96,5)\end{array}$ & & & & & & \\
\hline
\end{tabular}

FONTE: Coleta de dados, Feira de Santana - Bahia, 2015-2016.

${ }^{a}$ Em quatro CIP não foi possível visualizar essa variável

* Qui-quadrado de Pearson

** Teste exato de Fisher

\section{CONSIDERAÇÕES FINAIS}

Os resultados encontrados, neste estudo, registraram uma prevalência de 4,5\% de flebite na unidade em estudo, sendo considerado um nível aceitável pela INS. E mostrou que, 
em crianças com câncer, esta complicação associou-se de forma estatisticamente significante com a presença de tempo de hospitalização maior ou igual a 8 dias $(\mathrm{p}=0,022)$, TIV prolongada $(\mathrm{p}=0,000)$, calibre do cateter $22 \mathrm{G}(\mathrm{p}=0,025)$, tempo de utilização do $\mathrm{CIP} \geq 72$ horas $(\mathrm{p}=0,012)$ e tempo de utilização da terapia medicamentosa por maior ou igual 4 dias $(\mathrm{p}=0,019)$.

Este estudo traz uma valiosa contribuição e relevância profissional, teórica e social. Do ponto de vista profissional, os resultados poderão fornecer embasamento científico para que os profissionais de enfermagem atuantes em unidades oncológicas possam fundamentar sua prática clínica durante a realização da CIP em crianças e adolescentes hospitalizadas.

Para criança/adolescente os dados poderão potencializar a promoção de sua segurança durante a realização da TIV prescrita, reduzindo prováveis danos decorrentes da própria flebite e da realização de novas CIP. E ainda, contribuirá para o âmbito teórico, pois há uma escassez de publicações nacionais e internacionais sobre o objeto em estudo.

\section{REFERÊNCIAS}

ABDUL-HAK, C.K; BARROS, A.F. 2014. The incidence of phlebitis in a medical clinical unit.Texto contexto-enfermagem. 23 (3): 633-638.

BAGNASCO, A. et al. 2011. A Plan for the Prevention of Phlebitis in Paediatric Patients. Epeynhtikh eptazia. 74-53.

INFUSION NURSES SOCIETY (INS). 2016. Standards of Practice.J Infus Nurs., 39, (1S).

Infusion Nurses Society Brasil (INS - BRASIL). 2013. Diretrizes práticas para a terapia infusional. São Paulo.

JACINTO, A. K. L. et al.2014. Flebite associada a cateteres intravenosos periféricos em crianças: estudo de fatores predisponentes. Escola Anna Nery. 18(2): 220-226.

MAIA, V.R. et al. 2010. Protocolos de Enfermagem: Administração de quimioterapia antineoplásica no tratamento de hemopatias malignas. HEMORIO, $1^{\mathrm{a}}$ ed, p.38.

TRIPATHI, S; KAUSHIK, V; SINGH, V.K. 2008. Peripheral IVs: Factors affecting complications and patency-A randomized controlled trial. Journal Infusion Nurs. 3(31): 182188.

WEBSTER, J. e al. 2010. Clinically-indicated replacement versus routine replacement of peripheral venous catheters. Cochrane Database Systematic Reviews. v.17, n.3. 